\title{
M-QAM Modulation Symbol-Level Precoding for Power Minimization: Closed-Form Solution
}

\author{
Jevgenij Krivochiza, J.C. Merlano-Duncan, Symeon Chatzinotas, Björn Ottersten \\ SnT - securityandtrust.lu, University of Luxembourg, Luxembourg \\ Email: \{jevgenij.krivochiza, juan.duncan, symeon.chatzinotas, bjorn.ottersten\}@uni.lu
}

\begin{abstract}
In this paper, we derive a closed-form algorithm of the computationally efficient Symbol-Level Precoding (SLP) for power efficient communications when using $M$-QAM modulated waveforms. The channel state information (CSI) based and dataaided SLP technique optimizes power efficiency by solving a non-negative convex quadratic optimization problem per time frame of transmitted symbols. The optimization combines constructive inter-user interference to minimize the sum power of precoded symbols at the transmitter side under constraints for minimum SNR at the receiver side. The SLP implementation incurs extra computational complexity of the transmitter. We propose a convex quadratic optimization problem for $M$-QAM constellations and derive a closed-form algorithm with a fixed number of iterations to solve the problem.
\end{abstract}

Index Terms-MU-MISO, Interference Mitigation, Precoding, Beamforming, Power Minimization, Convex Optimization, NNLS, CVX, Computational Complexity.

\section{INTRODUCTION}

The upcoming fifth generation of cellular networks $(5 \mathrm{G})$ offers higher data rates and low latency communications [1], [2]. At the same time, building networks that support this growth of services have resulted in a corresponding rise in energy consumption. The new challenge will be to maintain the network's total energy consumption while introducing new features like increased data bandwidth and low latency [3]. Ericsson foresees that novel software features that can bring significant energy savings such as ultra-lean design and Massive Multiple-Input-Multiple-Output (MIMO) [4].

There are developed several precoding techniques to effectively manage and exploit MIMO wireless channels in the literature [5]. The most advanced techniques like symbollevel precoding (SLP) offer a variety of ways to improve MIMO communications [6]. In [7] we developed and demonstrated a novel SLP technique for sum power minimization at the transmitter side. The technique optimally preserves constructive interference, which leads to power minimization of the transmitted signal. Although, the technique supports optimization only of $M$-PSK and $M$-APSK symbols in the fixed-phase region.

Constructive interference (CI) vector perturbation (VP) based precoding [8] share the same approach as the technique developed in [7] to offer a reduction in algorithm complexity [9]. Other closed-form solutions were developed in [10][12] to further facilitate practical implementations of SLP techniques. Nevertheless, the main contribution of these works was in algorithmic complexity of SLP and CI-VP techniques, the practical implementation stayed out of their scope. In [13], [14] we demonstrated the feasibility to deliver low computationally complexity of SLP technique [7] and implement it on actual hardware processing the baseband in a real-time basis for a downlink transmission.

In this paper, we focus on terrestrial communication through a MU-MISO channel. We derive an energy and computationally efficient symbol-level precoding to minimize sum power of the transmitted signal while maintaining the minimal required SNR threshold at the receiver terminals. The SLP technique is based on a non-negative convex quadratic optimization problem. We improve the technique to support the optimization of $M$-th order QAM constellations in the relaxedphase region. In Section II we discuss the system model, define the optimization problem and propose a closed-form solution to solve it. In Section III we benchmark the performance of the SLP technique against Zero-Forcing precoding and compare the closed-form solution to CVX solver [15], [16]. Finally, we drew conclusions in Section IV.

Notation: Upper-case and lower-case bold-faced letters are used to denote matrices and column vectors. The superscripts $(\cdot)^{H},(\cdot)^{\dagger}$ and $(\cdot)^{-1}$ represents Hermitian matrix, matrix transpose and inverse operations. $\|\cdot\|_{2}$ is the Euclidean norm, $|\cdot|$ is an absolute magnitude of a complex value. The real and imaginary parts of a complex value are defined as $\operatorname{Re}(\cdot)$ and $\operatorname{Im}(\cdot)$. The imaginary unit is denoted as $\iota^{2}=-1$. Square diagonal matrices are denoted as $\operatorname{diag}[\cdot]$ with the elements defined on their main diagonal.

\section{Symbol-Level Precoding Design}

\section{A. System Model}

We consider a system model with the forward link of a multi-user multi-antenna terrestrial communication system. We assume the system to use the full frequency reuse scenario, in which all the antennas transmit in the same frequency and time. The multi-user interference is managed using precoding. We define the number of transmitting antenna as $N_{t}$ and the total number of receiver terminals as $N_{u}$ in the coverage area. In the specified MU-MISO channel model, the received signal at the $\mathbf{i}$-th terminal is given by $y_{i}=\mathbf{h}_{i}^{\dagger} \mathbf{x}+n_{i}$, where $\mathbf{h}_{i}^{\dagger}$ is a $1 \times N_{t}$ vector representing the complex channel coefficients between the $i$-th terminal and the $N_{t}$ antennas of the transmitter, $\mathbf{x}$ is defined as the $N_{t} \times 1$ vector of the transmitted symbols 
at a certain symbol period and $n_{i}$ is the independent complex circular symmetric (c.c.s.) independent identically distributed (i.i.d) zero mean Additive White Gaussian Noise (AWGN) measured at the $i$-th terminal's receive antenna.

Looking at the concatenated formulation of the received signal, which includes the whole set of receiver terminals, the linear signal model is

$$
\mathbf{y}=\mathbf{H x}+\mathbf{n}=\mathbf{H W} \mathbf{s}+\mathbf{n},
$$

where $\mathbf{y}=\left[y_{1}, y_{2}, \ldots, y_{i}\right] \in \mathbb{C}^{N_{u} \times 1}, \mathbf{n}=\left[n_{1}, n_{2}, \ldots, n_{i}\right] \in$ $\mathbb{C}^{N_{u} \times 1}, \mathbf{x} \in \mathbb{C}^{N_{t} \times 1}$, and $\mathbf{s} \in \mathbb{C}^{N_{u} \times 1}$ and $\mathbf{H}=$ $\left[\mathbf{h}_{i}^{\dagger}, \mathbf{h}_{i}^{\dagger}, \ldots, \mathbf{h}_{i}^{\dagger}\right] \in \mathbb{C}^{N_{u} \times N_{t}}$. In this scenario, we define a precoding matrix $\mathbf{W} \in \mathbb{C}^{N_{t} \times N_{u}}$ which maps the information symbols $\mathbf{s}$ into precoded symbols $\mathbf{x}$. We consider the data symbols $\mathbf{s}$ to be unit variance complex vectors $\left|s_{i}\right|=1$ for every $i=1,2, \ldots, N_{u}$.

\section{B. Symbol-Level Precoding Design}

In this section, we define an optimization problem of the computationally efficient SLP technique, which aims to minimize the sum power of the precoded symbols at the gateway side. The technique is applicable on the $M$-th order quadrature amplitude modulation ( $M$-QAM). It reduces the sum power of the precoded symbols by optimally increasing the amplitudes of the initial data symbols to exploit the constructive interference at the receiver side. The method optimally preserves constructive interference components to decrease the total transmitted power at the transmitter side. To achieve this, the SLP technique needs to recalculate a perturbation vector $\mathbf{u}=\left[u_{1}, u_{2}, \ldots, u_{i}\right] \in \mathbb{C}^{N_{u} \times 1}$ for a set of symbols $\mathbf{s}$ in every transmission time frame to construct the optimized precoded signal given by

$$
\mathbf{x}=\mathbf{W} \boldsymbol{\Gamma}(\mathbf{s}+\mathbf{u}),
$$

where $\boldsymbol{\Gamma}$ is per terminal minimal SNR requirements defined as

$$
\boldsymbol{\Gamma}=\operatorname{diag}\left[\Gamma_{1}, \quad \Gamma_{2}, \ldots, \quad \Gamma_{i}\right] .
$$

In the following formulation, we split the problem of constructing optimal beamforming vectors into two independent tasks: channel orthogonalization and optimal symbol mapping for energy efficiency. In this case, we define the precoding matrix $(\mathbf{W})$ as the Zero-Forcing linear precoder:

$$
\mathbf{W}_{\mathrm{ZF}}=\hat{\mathbf{H}}^{H}\left(\hat{\mathbf{H}} \hat{\mathbf{H}}^{H}\right)^{-1},
$$

where $\hat{\mathbf{H}}$ is the channel matrix estimated from the channel state information (CSI). We choose $\mathrm{ZF}$ for its properties to orthogonalize the channel so that in the case of $\hat{\mathbf{H}}=\mathbf{H}$ the SNRs of the received symbols are linearly related to the $\Gamma$ applied on the transmitted symbols, the perturbation vector (u) and the Gaussian noise (n):

$$
\mathbf{y}=\mathbf{H W}_{\mathrm{ZF}} \boldsymbol{\Gamma}(\mathbf{s}+\mathbf{u})+\mathbf{n}=\boldsymbol{\Gamma} \mathbf{s}+\boldsymbol{\Gamma} \mathbf{u}+\mathbf{n} .
$$

Thought, ZF is not an optimal precoder in a sense of energy efficiency and there are more efficient techniques in the literature [17], which provide better performance on a frame
TABLE I

PROPERTIES OF PERTURBATION VECTOR BASED ON SYMBOL POSITION

\begin{tabular}{|c||c|c|c|c|}
\hline Position & $\operatorname{Re}\left(\tilde{s}_{i}\right)$ & $\operatorname{Im}\left(\tilde{s}_{i}\right)$ & $\operatorname{Re}\left(u_{i}\right)$ & $\operatorname{Im}\left(u_{i}\right)$ \\
\hline$p_{1}$ & $\geq 1$ & $\geq 1$ & $\geq 0$ & $\geq 0$ \\
$p_{2}$ & $\geq 1$ & $<1$ & $\geq 0$ & $=0$ \\
$p_{3}$ & $<1$ & $\geq 1$ & $=0$ & $\geq 0$ \\
$p_{4}$ & $<1$ & $<1$ & $=0$ & $=0$ \\
\hline
\end{tabular}

basis. The proposed SLP approach will build upon the ZF precoder and will improve the energy efficiency by optimizing every transmitted set of symbols. The optimal symbol mapping we derive in the following paragraphs.

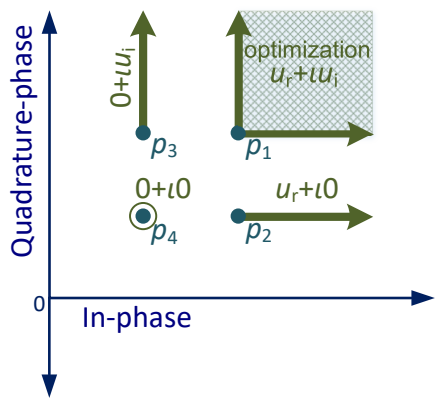

Fig. 1. 16-QAM constellation optimization with the proposed SLP technique

In Fig. 1 we demonstrate an impact of the perturbation $u_{i}$ on a complex data symbol $s_{i}$ depending on its location in the $M$-QAM constellation. The perturbation vector has to increase the absolute magnitude of the symbol and preserve detection regions. We consider that all the symbols are mapped in the first quadrature of the complex plane by transforming the actual complex data symbols with the equivalent symbols $\tilde{\mathbf{s}} \in \mathbb{C}^{N_{u} \times 1}$, where $\tilde{s}_{i}=b_{i} s_{i}, \operatorname{Re}\left(\tilde{s}_{i}\right) \geq 0$ and $\operatorname{Im}\left(\tilde{s}_{i}\right) \geq 0$ for every $i=1,2, \ldots, N_{u}$. The variable $b_{i}$ represents the rotation of the symbol vectors into the first quadrature of the complex plane and is defined as

$$
b_{i}=\left\{\begin{array}{ll}
1 & \text { if } \operatorname{Re}\left(s_{i}\right)>0 \text { and } \operatorname{Im}\left(s_{i}\right)>0 \\
\iota 1 & \text { if } \operatorname{Re}\left(s_{i}\right)<0 \text { and } \operatorname{Im}\left(s_{i}\right)>0 \\
-\iota 1 & \text { if } \operatorname{Re}\left(s_{i}\right)>0 \text { and } \operatorname{Im}\left(s_{i}\right)<0 \\
-1 & \text { if } \operatorname{Re}\left(s_{i}\right)<0 \text { and } \operatorname{Im}\left(s_{i}\right)<0
\end{array},\right.
$$

for $i=1,2, \ldots, N_{u}$. The following equality is therefore respected

$$
\mathbf{s}=\mathbf{B} \tilde{\mathbf{s}}
$$

where $\mathbf{B}$ is a diagonal matrix, where the rotation vectors $b_{i}$ are its diagonal elements such as:

$$
\mathbf{B}=\operatorname{diag}\left[b_{1}, \quad b_{2}, \quad b_{3}, \ldots, b_{i}\right] .
$$

We identify 4 possible positions of a symbol on the complex plain regardless of the modulation order $M$ considered and summarize the constraints on the perturbation vector based on the position of a symbol in Table I. We replace the perturbation vector $\mathbf{u}$ with a new vector $\tilde{\mathbf{u}}=\left[\tilde{u_{1}}, \tilde{u_{2}}, \ldots, \tilde{u_{i}}\right] \in \mathbb{C}^{N_{u} \times 1}$, 
where $\operatorname{Re}\left(\tilde{u}_{i}\right) \geq 0$ and $\operatorname{Im}\left(\tilde{u}_{i}\right) \geq 0$ for every $i=1,2, \ldots, N_{u}$. Finally, we rewrite the equation (2) as

$$
\mathbf{x}=\mathbf{W}_{\mathrm{ZF}} \boldsymbol{\Gamma B}(\tilde{\mathbf{s}}+\tilde{\mathbf{u}}) .
$$

We define the optimization problem to minimize the sum power of the precoded symbols vector $\mathrm{x}$ for any $M$-QAM constellation as

$$
\begin{array}{ll}
\min _{\tilde{\mathbf{u}}} & \|\mathbf{x}\|_{2} \\
\text { s. t. } \quad C 1:\left\{\begin{array}{l}
\operatorname{Re}\left(\tilde{u}_{i}\right) \geq 0 \\
\operatorname{Im}\left(\tilde{u}_{i}\right) \geq 0
\end{array}, \quad \tilde{s}_{i} \in p_{1} .\right.
\end{array}
$$

for all $i=1,2, \ldots, N_{u}$.

By substituting (9) in (10) and by applying an equality between the Euclidean norm of a complex vector $\tilde{z}=$ $\left[\tilde{z_{1}}, \tilde{z_{2}}, \ldots, \tilde{z_{i}}\right]$ and a real vector $z=\left[z_{1}, z_{2}, \ldots, z_{i}\right]$, where $\tilde{z_{i}}=a_{i}+\iota b_{i}$ and $z_{i}=\left[a_{i}, b_{i}\right]$, we get

$$
\begin{aligned}
& \min _{\hat{\mathbf{u}}}\|\hat{\mathbf{A}} \hat{\mathbf{u}}-\hat{\mathbf{d}}\|_{2} \\
& \text { s. t. } \quad C 1:\left\{\begin{array}{l}
\hat{u}_{i} \geq 0 \\
\hat{u}_{i+N_{u}} \geq 0
\end{array}, \quad \tilde{s}_{i} \in p_{1}\right. \text {. } \\
& C 2:\left\{\begin{array}{l}
\hat{u}_{i} \geq 0 \\
\hat{u}_{i+N_{u}}=0
\end{array}, \quad \tilde{s}_{i} \in p_{2} .\right. \\
& C 3:\left\{\begin{array}{l}
\hat{u}_{i}=0 \\
\hat{u}_{i+N_{u}} \geq 0
\end{array}, \quad \tilde{s}_{i} \in p_{3} .\right. \\
& C 4:\left\{\begin{array}{l}
\hat{u}_{i}=0 \\
\hat{u}_{i+N_{u}}=0
\end{array}, \quad \tilde{s}_{i} \in p_{4},\right.
\end{aligned}
$$

where $\widehat{\mathbf{A}}=[\operatorname{Re}(\mathbf{A}),-\operatorname{Im}(\mathbf{A}) ; \operatorname{Im}(\mathbf{A}), \operatorname{Re}(\mathbf{A})] \in \mathbb{R}^{2 N_{t} \times 2 N_{u}}$, $\hat{\mathbf{d}}=\left[\operatorname{Re}\left(\mathbf{d}^{\dagger}\right), \operatorname{Im}\left(\mathbf{d}^{\dagger}\right)\right]^{\dagger} \in \mathbb{R}^{2 N_{t} \times 1}, \hat{\mathbf{u}}=\left[\operatorname{Re}\left(\tilde{\mathbf{u}}^{\dagger}\right), \operatorname{Im}\left(\tilde{\mathbf{u}}^{\dagger}\right)\right]^{\dagger} \in$ $\mathbb{R}^{2 N_{u} \times 1}, \mathbf{A}=\mathbf{W}_{\mathrm{ZF}} \boldsymbol{\Gamma B}$, and $\mathbf{d}=-\mathbf{W}_{\mathrm{ZF}} \mathbf{B} \tilde{\mathbf{s}}$. In general, the optimization problem (11) can be solved using CVX tools for MATLAB [15], [16]. In the next section, we propose a closedform algorithm to solve the problem with a fixed number of iterations.

\section{Closed-form Algorithm}

To derive the closed-form algorithm to solve the optimization problem (11), we firstly address only the constraint $C 1$ of the problem and then expand the approach to deal with the constraints $C 1, C 2, C 3$ and $C 4$ jointly. The optimization problem (11) with the constraint $C 1$ has a form of a nonnegative least squares (NNLS) problem. It can be solved using iterative Fast NNLS algorithm [18], [19].
The conventional Fast NNLS algorithm finds the optimal regression coefficients through a number of iteration. In every iteration, it dynamically chooses and solves a subset of quadratic equations from a complete set defined as

$$
\hat{\mathbf{u}}=\left(\widehat{\mathbf{A}}^{\dagger} \widehat{\mathbf{A}}\right)^{-1} \widehat{\mathbf{A}}^{\dagger} \hat{\mathbf{d}} \text {. }
$$

For Fast NNLS algorithm to converge the number of iteration is not fixed and can reach up to $N_{u}$. In every iteration, the equation (12) is solved by using the $\mathrm{QR}$ decomposition, which asymptotic complexity alone is of $O\left(N_{t} \times N_{u}^{2}\right)$.

We propose to substantially relax the complexity of the optimization problem by the assumption that the regression coefficients are mutually uncorrelated. In this case, the offdiagonal elements of the matrix product $\left(\widehat{\mathbf{A}}^{\dagger} \widehat{\mathbf{A}}\right)$ are equal to zero as

$$
\widehat{\mathbf{A}}^{\dagger} \widehat{\mathbf{A}} \approx\left[\begin{array}{ccc}
\sum_{j=1}^{2 N_{t}} \widehat{A}_{j, 1}^{2} & \cdots & 0 \\
\vdots & \ddots & \vdots \\
0 & \cdots & \sum_{j=1}^{2 N_{t}} \widehat{A}_{j, 2 N_{u}}^{2}
\end{array}\right] .
$$

By inserting (13) into (12) we derive an approximate closedform solution to calculate the perturbation vector for the optimization problem (11) as

$$
\hat{u}_{k}=\frac{1}{\sum_{j=1}^{2 N_{t}} \widehat{A}_{j, k}^{2}} \sum_{j=1}^{2 N_{t}} \widehat{A}_{j, k} \hat{d}_{j} \geq 0,
$$

for each $k=1, \ldots, 2 N_{u}$ of the vector $\hat{\mathbf{u}}$. The solution of the equation (14) must be equal or greater than zero and cannot take negative values.

We extend the solution derived for the constraint $C 1$ to also meet the requirements of $C 2, C 3, C 4$. We can deduct a new rule from Figure 1 and Table I saying that if a real or imaginary part of a symbol is mapped on the top level of the constellation $\left(\operatorname{Re}\left(\tilde{s}_{i}\right) \geq 1\right.$ or $\left.\operatorname{Im}\left(\tilde{s}_{i}\right) \geq 1\right)$ this part can be increased accordingly to the optimal solution. In other cases, the symbol should remain in its original position. We rewrite the rule in an equation form as follows:

$$
\hat{u}_{k}= \begin{cases}\frac{1}{\sum_{j=1}^{2 N_{t} \widehat{A}_{j, k}^{2}} \sum_{j=1}^{2 N_{t}} \widehat{A}_{j, k} \hat{d}_{j} \geq 0} & , \text { if } \hat{s}_{i} \geq 1 \\ 0 & , \text { if } \hat{s}_{i}<1\end{cases}
$$

where $\hat{\mathbf{s}}=\left[\operatorname{Re}\left(\tilde{\mathbf{s}}^{\dagger}\right), \operatorname{Im}\left(\tilde{\mathbf{s}}^{\dagger}\right)\right]^{\dagger} \in \mathbb{R}^{2 N_{u} \times 1}$.

We can rewrite equation (15) in an algorithmic form to provide some insights on its computational complexity. We can see that Algorithm 1 has a fixed number of iterations and that asymptotic complexity of the complete closed-form solution is of $O\left(N_{t} \times N_{u}\right)$.

\section{NumericAl RESUlts}

To generate numerical results, we consider a MU-MISO system, which has an equal number of the transmit and receive antennas $N_{t}=N_{u}=N$. We accordingly generate a full rank $N \times N$ MU-MISO channel matrix with i.i.d. complex values with a 2-norm matrix condition number defined as

$$
\kappa_{2}(\mathbf{H})=\|\mathbf{H}\|_{2} \cdot\left\|\mathbf{H}^{-\mathbf{1}}\right\|_{2} .
$$




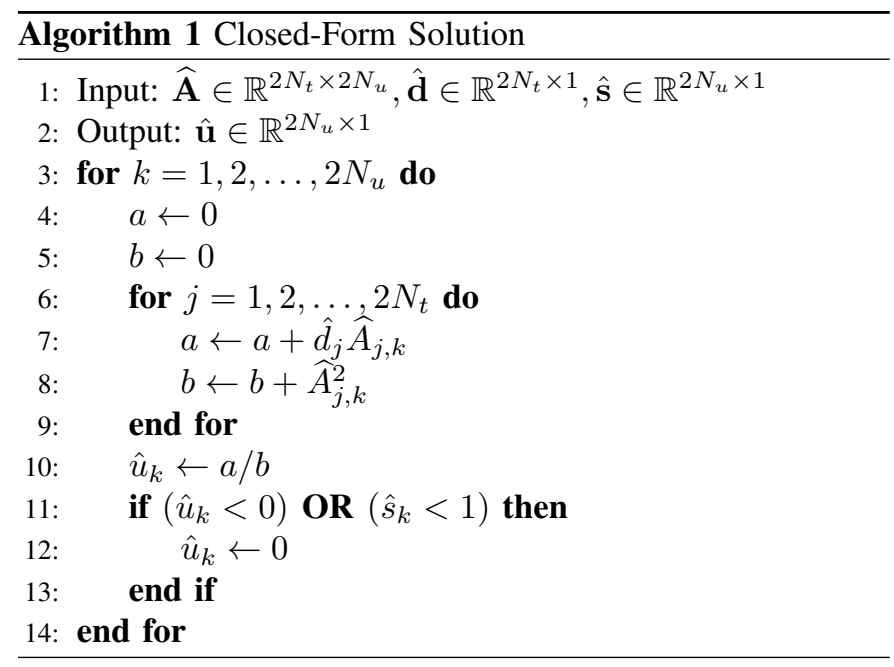

The matrix condition number corresponds to the ratio of the largest singular value of that matrix to the smallest singular value. In the case of the MU-MISO system, the matrix condition number describes the power imbalance in the channel. To generate a channel matrix with random i.i.d. values and a desired condition number we calculate singularvalue decomposition (SVD) [20] of the generated matrix (H) as $\mathbf{H}=\mathbf{U} \boldsymbol{\Sigma} \mathbf{V}^{\dagger}$. $\mathbf{U}$ is an $N_{u} \times N_{u}$ complex unitary matrix, $\boldsymbol{\Sigma}$ is an $N_{u} \times N_{t}$ rectangular diagonal matrix with non-negative real numbers on the diagonal, and $\mathbf{V}$ is an $N_{t} \times N_{t}$ real or complex unitary matrix. We reconstruct $\boldsymbol{\Sigma}$ for its diagonal elements to monotonically increase from 1 to $\kappa_{2}$ as

$$
\Sigma_{\kappa_{2}}=\operatorname{diag}\left[1, \ldots, \kappa_{2}\right] .
$$

The resulting channel matrix is then computed and its power is normalized as

$$
\mathbf{H}=\frac{\mathbf{U} \boldsymbol{\Sigma}_{\kappa_{2}} \mathbf{V}^{\dagger}}{\sqrt{\sum_{n=1}^{N_{u}} \sum_{m=1}^{N_{t}} h_{n, m} h_{n, m}^{H}}} .
$$

We average the results over 100 channel matrix iterations with defined condition number $\left(\kappa_{2}\right)$. We benchmark the proposed approximate closed-form algorithm and CVX optimization algorithms and measure the total average power of the precoded symbols generated by the techniques in selected channel scenarios. We set the minimal SNR requirement to $12 \mathrm{~dB}\left(\Gamma_{i}=10^{12 / 10}\right)$ for every $i=1,2, \ldots, N_{u}$. We chose the modulation order $M=16$ to conduct all the benchmarks.

\section{A. Transmitted Signal Power}

In Fig. 2 we see the total average power of the approximate closed-form and CVX optimization algorithms. The power of the precoded symbols generated by ZF precoder is present as a baseline for the benchmarks. This way we can directly compare the increase in the performance of the techniques under the same conditions. The condition number of the channel matrices used in the benchmarks is set as a function of $3 N$. For example, for 10 antennas at the transmitter, the condition number of all the $10 \times 10$ channel matrices is 30 ,

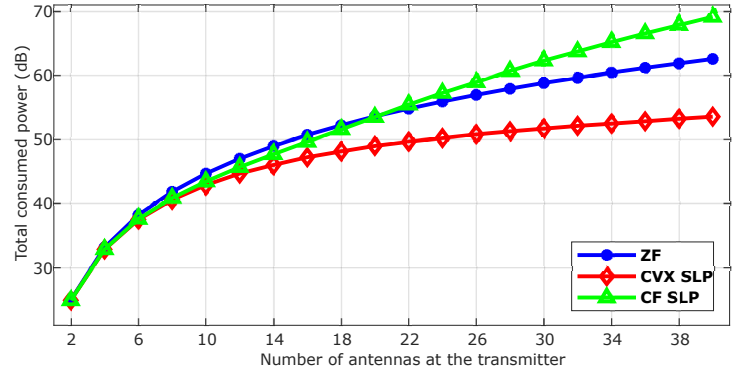

Fig. 2. Total power of the transmitted precoded symbols calculated by ZF, the proposed close-form and CVX algorithms.

for 20 antennas the condition is 60 , and for 40 antennas the condition number is 120 . The impact of the condition number on the solution of the proposed closed-form algorithm was demonstrated in [7]. In this benchmark, we choose these values to demonstrate the representative behavior of the algorithm. The approximate closed-form algorithm (CF SLP) performs very closely to the CVX (CVX SLP) approach up to certain dimensions of the channel matrices. We can see that after the certain size $N$ of the MU-MISO system the CVX substantially outperforms the proposed closed-form algorithm in these cases. Moreover, the proposed closed-form algorithm is performing at higher power than the baseline $\mathrm{ZF}$ technique for $N>20$ region. It is evident, that the proposed closed-form algorithm, which is based on certain assumptions discussed in Section II-C, performs with lower power consumption than ZF for small scale MU-MISO systems $N \leq 20$. The performance is also fairly close to the performance of CVX algorithm for systems with $N<10$. For larger systems with $N>20$, the power consumption of the closed-form algorithm appears to be higher than both ZF and CVX approaches.

\section{B. Received Signal SNR}

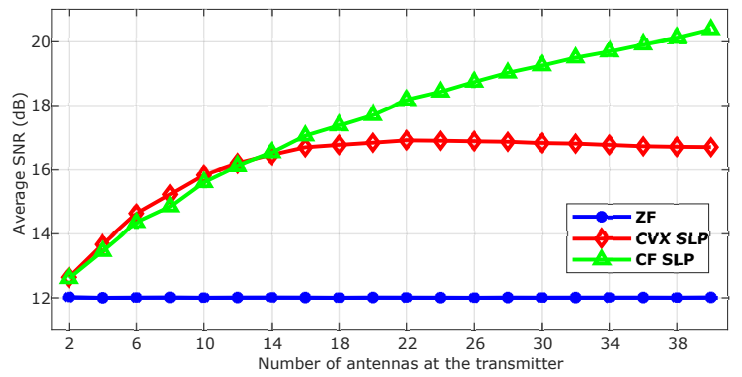

Fig. 3. Average SNR per UT of the received precoded symbols calculated by $\mathrm{ZF}$, the proposed close-form and CVX algorithms.

In Fig. 3 we observe the averaged SNR values at the receive terminals, those correspond to the consumed power at the transmitter side shown in Fig. 2. The SNR at the $i$-th terminal is calculated as

$$
\mathrm{SNR}_{i}=10 \log _{10}\left(\frac{\left\|\mathbf{h}_{i}^{\dagger} \mathbf{w}_{i} \Gamma_{i} b_{i}\left(\tilde{s}_{i}+\tilde{u}_{i}\right)\right\|_{2}}{\left\|n_{i}\right\|_{2}}\right),
$$

where $\mathbf{w}_{i}$ - is the $i$-th row of the ZF matrix (4). The proposed closed-form algorithm provides higher SNR for terminals than 
$\mathrm{ZF}$ in the whole benchmark region due to the perturbation vector $\tilde{\mathbf{u}}$ is positively contributing to the power of the symbols. Notably, the proposed algorithm also outperforms even CVX optimization in the delivered SNR for large scale MU-MISO systems $N>14$.

\section{Signal Processing Time}

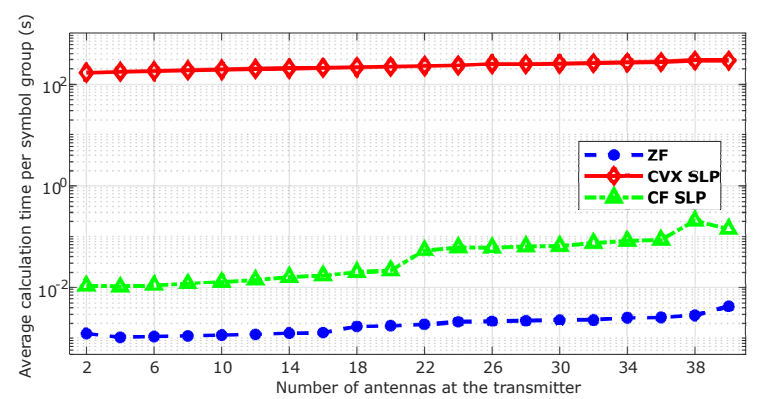

Fig. 4. Average processing time of the precoded symbols calculated by ZF, the proposed close-form and CVX algorithms.

The following benchmark shows the average processing time per set of $N$ precoded symbols. The benchmark was launched in the MATLAB R2018b environment using a general-purpose central processing unit. Fig. 4 shows the time required to process for $\mathrm{ZF}$, the proposed closed-form and CVX algorithms. The ZF and CF SLP algorithms perform much faster in the conducted benchmark than CVX. The significant improvement of the processing time is a considerable advantage for the closed-form algorithm over more efficient CVX technique. The algorithm potentially allows implementing symbol-level precoding for an advanced energy efficient interference mitigation in novel wireless communications.

\section{CONCLUSIONS}

In this paper, we presented the closed-form algorithm to solve a convex quadratic optimization problem for sum power minimization in the energy efficient symbol-level precoding. We derive the optimization problem for sum power minimization of symbols in $M$-QAM constellation and propose a closed-form algorithm to solve this problem with low computational complexity. We demonstrated with numerical benchmarks the efficiency of the precoder design solved by CVX and the proposed closed-form algorithm. The numerical results demonstrated the feasibility of the closed-form algorithm to reduce sum power up to a certain MU-MISO system parameter $N$. The proposed SLP design allows delivering high SNR at the receive terminals even if the closed-form algorithm is not performing as well as CVX approach power minimization wise. The developed closed-form algorithm provides a tradeoff between the lower power optimization and much faster processing time. The efficient processing time and low complexity of the algorithm are important for the realistic implementation of precoding for energy efficient wireless communications.

\section{ACKNOWLEDGMENT}

This research was supported by Luxembourg National Research Fund grant FNR11481283 "End-to-end Signal Processing Algorithms for Precoded Satellite Communications".

\section{REFERENCES}

[1] Y. B. Zikria, S. W. Kim, M. K. Afzal, H. Wang, and M. H. Rehmani, "5G Mobile Services and Scenarios: Challenges and Solutions," Sustainability, vol. 10, no. 10, 2018.

[2] G. Liu and D. Jiang, "5G: Vision and Requirements for Mobile Communication System towards Year 2020," Chinese Journal of Engineering, vol. 2016, p. 8, 2016.

[3] I. B. Sofi and A. Gupta, "A Survey on Energy Efficient 5G Green Network with a Planned Multi-Tier Architecture," Journal of Network and Computer Applications, vol. 118, pp. 1 - 28, 2018.

[4] B. Joshi. (2019) Breaking the Energy Curve: Why Service Providers Should Care About 5G Energy Efficiency. [Online]. Available: https://www.ericsson.com/en/blog/2019/ 2/breaking-the-energy-curve-5g-energy-efficiency

[5] M. Alodeh, D. Spano, A. Kalantari, C. G. Tsinos, D. Christopoulos, S. Chatzinotas, and B. Ottersten, "Symbol-Level and Multicast Precoding for Multiuser Multiantenna Downlink: A State-of-the-Art, Classification, and Challenges," IEEE Communications Surveys Tutorials, vol. 20, no. 3, pp. 1733-1757, thirdquarter 2018.

[6] M. Alodeh, S. Chatzinotas, and B. Ottersten, "Symbol-Level Multiuser MISO Precoding for Multi-Level Adaptive Modulation," IEEE Transactions on Wireless Communications, vol. 16, no. 8, pp. 5511-5524, Aug 2017.

[7] J. Krivochiza, J. C. Merlano-Duncan, S. Andrenacci, S. Chatzinotas, and B. Ottersten, "Closed-Form Solution for Computationally Efficient Symbol-Level Precoding," in 2018 IEEE Global Communications Conference (GLOBECOM), Dec 2018, pp. 1-6.

[8] B. M. Hochwald, C. B. Peel, and A. L. Swindlehurst, "A vector-perturbation technique for near-capacity multiantenna multiuser communication-part ii: perturbation," IEEE Transactions on Communications, vol. 53, no. 3, pp. 537-544, March 2005.

[9] C. Masouros, M. Sellathurai, and T. Ratnarajah, "Vector perturbation based on symbol scaling for limited feedback miso downlinks," IEEE Transactions on Signal Processing, vol. 62, no. 3, pp. 562-571, Feb 2014.

[10] A. Li and C. Masouros, "Interference Exploitation Precoding Made Practical: Optimal Closed-Form Solutions for PSK Modulations," IEEE Transactions on Wireless Communications, vol. 17, no. 11, pp. 76617676,2018

[11] A. Haqiqatnejad, F. Kayhan, and B. Ottersten, "Power Minimizer Symbol-Level Precoding: A Closed-Form Suboptimal Solution," IEEE Signal Processing Letters, vol. 25, no. 11, pp. 1730-1734, Nov 2018.

[12] S. Domouchtsidis, C. G. Tsinos, S. Chatzinotas, and B. Ottersten, "Symbol-level precoding for low complexity transmitter architectures in large-scale antenna array systems," IEEE Transactions on Wireless Communications, vol. 18, no. 2, pp. 852-863, Feb 2019.

[13] J. Krivochiza, J. Merlano Duncan, S. Andrenacci, S. Chatzinotas, and B. Ottersten, "FPGA Acceleration for Computationally Efficient Symbol-Level Precoding in Multi-User Multi-Antenna Communication Systems," IEEE Access, vol. 7, pp. 15 509-15 520, 2019.

[14] J. Krivochiza, J. C. Merlano-Duncan, S. Andrenacci, S. Chatzinotas, and B. Ottersten, "Computationally and Energy Efficient Symbol-Level Precoding Communications Demonstrator," Physical Communication, vol. 28, pp. $108-115,2018$.

[15] M. Grant and S. Boyd, "CVX: Matlab Software for Disciplined Convex Programming, version 2.1," http://cvxr.com/cvx, Mar. 2014.

[16] _ "Graph implementations for nonsmooth convex programs," in Recent Advances in Learning and Control, ser. Lecture Notes in Control and Information Sciences, V. Blondel, S. Boyd, and H. Kimura, Eds. Springer-Verlag Limited, 2008, pp. 95-110, http://stanford.edu/ boyd/ graph_dcp.html.

[17] M. Alodeh, S. Chatzinotas, and B. Ottersten, "Constructive Multiuser Interference in Symbol Level Precoding for the MISO Downlink Channel," IEEE Transactions on Signal Processing, vol. 63, no. 9, pp. 2239-2252, May 2015.

[18] C. L. Lawson, . Hanson, Richard J., S. for Industrial, and A. Mathematics, Solving least squares problems, [rev. ed.] ed. Philadelphia : SIAM, 1995.

[19] R. Bro and S. De Jong, "A Fast Non-negativity-constrained Least Squares Algorithm," Journal of Chemometrics, vol. 11, no. 5, pp. 393401, 1997.

[20] S. Banerjee, Linear Algebra and Matrix Analysis for Statistics (Texts in Statistical Science). Chapman and Hall, jun 2014. 